\title{
Nefropatía por aciclovir en un paciente con sospecha de toxicidad por opioides: caso clínico
}

\author{
C. Guerrero ${ }^{1}$, J. Moyano ${ }^{1}$, M. F. Ulloa 2 , S. Pacheco ${ }^{3}$ y P. Chinchilla ${ }^{4}$ \\ ${ }^{1}$ Departamento de Anestesiología y Clínica de Dolor. Hospital Universitario Fundación Santafé de Bogotá. \\ Colombia. ${ }^{2}$ Servicio de Anestesiología y Reanimación. Hospital Universitario Fundación Cardioinfantil. \\ Colombia ${ }^{3}$ Servicio de Anestesiología y Reanimación. Hospital Universitario Fundación Santafé de Bogotá. \\ Colombia. ${ }^{4}$ Servicio de Anestesiología y Reanimación. Hospital Occidente de Kennedy. Bogotá, Colombia
}

Guerrero C, Moyano J, Ulloa MF, Pacheco S, Chinchilla $P$. Nefropatía por aciclovir en un paciente con sospecha de toxicidad por opioides: caso clínico. Rev Soc Esp Dolor 2015; 22(1): 36-38.

\begin{abstract}
Based on the evidence, the current management of herpes zoster and its sequela post-herpetic neuralgia $(\mathrm{PHN})$ relies upon the early onset of antiviral agents and analgesics, and depending on the severity of the pain those can be strong opioids. It is uncommon to monitor the renal function in patients with this treatment, even though there are reports of nephrotoxicity secondary to the antiviral use. We presented a case of a 49-year-old woman who was admitted for herpes zoster treatment, and developed renal insufficiency following a treatment with acyclovir. The main clinical manifestation was a change in the neurologic status, which was first interpreted as opioid toxicity. The patient was treated with hemodialysis obtaining full recovery. We consider this report relevant as it stresses the importance of monitoring the renal function in patients receiving this type of medication.
\end{abstract}

Key words: Acyclovir. Acute renal failure. Herpes zoster. Neuralgia. Opioids. Opioid toxicity.

\section{RESUMEN}

El tratamiento actual de la neuritis herpética, basado en la evidencia, comprende la administración precoz de antivirales y analgésicos que según su intensidad pueden ser opioides potentes. No es común realizar en forma rutinaria la monitorización de la función renal a pesar de que existen reportes sobre insuficiencia renal secundaria al uso de antivirales. Presentamos un caso de nefrotoxicidad por aciclovir, en donde la manifestación clínica principal fue la alteración del estado de consciencia que fue interpretado como toxicidad por opioides. Se trata de una paciente femenina de 49 años con cuadro de neuritis herpética en tratamiento analgésico con morfina y codeína, quien desarrolló deterioro agudo del estado de consciencia e insuficiencia renal aguda, requiriendo hemodiálisis. Consideramos relevante este caso, con la intención de resaltar la importancia de la monitorización de la función renal en los pacientes que reciben terapia con estos medicamentos.

Palabras clave: Aciclovir. Insuficiencia renal aguda. Herpes zóster. Neuralgia. Opioides. Neurotoxicidad.

\section{INTRODUCCIÓN}

Las guías de manejo del dolor neuropático realizadas por diversas organizaciones como el Grupo de Interés Especial en Dolor Neuropático, de la Asociación Internacional para el Estudio del Dolor, la Sociedad Europea para el Manejo del Dolor Neuropático y otras recomiendan utilizar neuromoduladores, opioides y antivirales en los pacientes con dolor por herpes zóster. Presentamos el caso clínico de una paciente con herpes zóster sin enfermedad renal previa, en manejo con morfina y codeína, que desarrolló insuficiencia renal aguda secundaria a administración de aciclovir. Las manifestaciones neurológicas iniciales fueron interpretadas como toxicidad por opioides enmascarando la verdadera etiología del cuadro. 


\section{CASO CLÍNICO}

Paciente femenina de 49 años (peso: 60 kilos; talla: 1,63 metros) quien consulta al servicio de urgencias por cuadro de 5 días de dolor lumbar izquierdo, tipo ardor y eléctrico, de intensidad 10/10 en escala verbal análoga. El dolor es episódico, exacerbado con el movimiento y no mejora con la administración de tramadol y diclofenaco. Al examen físico se encuentran lesiones eritematosas, vesiculares con distribución dermatómica en la piel de la región lumbar y glúteo izquierdo. El resto del examen físico es normal. Presenta antecedentes de discopatía lumbar, hipertensión arterial e hipotiroidismo en tratamiento médico. La paciente niega antecedente de enfermedad renal.

La paciente es valorada por servicio de infectología quienes consideran que se trata de un cuadro de neuritis herpética y deciden, por la severidad del cuadro, hospitalizar a la paciente. Durante el primer día y previo al protocolo de hidratación ( $500 \mathrm{ml}$ de solución salina $0,9 \%$ ) se inicia infusión de aciclovir intravenoso $10 \mathrm{mg} / \mathrm{kg}$ cada 8 h (600 mg). Se solicita interconsulta a clínica de dolor quienes inician esquema analgésico con acetaminofén + codeína $(500+$ $30 \mathrm{mg}$ vía oral cada $6 \mathrm{~h}$ ), dosis de morfina en caso de dolor severo ( $2 \mathrm{mg}$ por dosis) y pregabalina $(150 \mathrm{mg} / 12 \mathrm{~h})$. La paciente presenta, inicialmente, adecuada analgesia sin efectos secundarios, calificando su dolor en 4/10.

Durante el segundo día de hospitalización presenta mareo, náuseas, emesis, somnolencia y disartria. Se considera que el cuadro es un efecto secundario a los analgésicos y se decide suspender el acetaminofén con codeína y la pregabalina. Se prescriben dosis de morfina de $2 \mathrm{mg}$ en caso de dolor severo previa evaluación de estado de consciencia. En el tercer día de hospitalización, sin recibir ninguna dosis de opioide, la paciente persiste con deterioro de su estado general, con progresión a estupor, taquipnea, desaturación y dificultad respiratoria. Se traslada a Unidad de Cuidados Intensivos. El cuadro es compatible con un edema pulmonar agudo y una insuficiencia renal con potasio sérico en $5,7 \mathrm{mEq} / \mathrm{L}$, BUN $50 \mathrm{mg} / \mathrm{dl}$ (valor de ingreso de 17,2), creatinina $5,01 \mathrm{mg} / \mathrm{dl}$ (valor de ingreso de 0,66 ) y gases arteriales con acidosis metabólica. Nefrología confirma diagnóstico de insuficiencia renal aguda secundaria a administración de aciclovir, con necesidad de hemodiálisis. La paciente requirió sesión única de hemodiálisis con mejoría en los niveles de azoados. Se continúa manejo analgésico con acetaminofén 500 mg y codeína $30 \mathrm{mg}$; la paciente no requiere nuevas dosis de morfina. Egresa al sexto día de hospitalización con dolor controlado y con pruebas de función renal normales.

\section{DISCUSIÓN}

Existen varios reportes de insuficiencia renal aguda por aciclovir descritos en la literatura, tanto para la población infantil como la adulta (1). El mecanismo fisiopatológico se relaciona con cristalización de la droga en los túbulos renales $(2,4)$ basados en los hallazgos de cristaluria en varios reportes de casos y al menos un reporte prospectivo. Se han descrito factores de riesgo para su desarrollo tales como altas dosis del antiviral, uso concomitante de medicamentos nefrotóxicos, insuficiencia renal previa, edad avanzada y estado de deshidratación (5). Sin embargo, cada vez es más frecuente el reporte de casos en pacientes previamente sanos (3). Generalmente se resuelve varios días después de la suspensión del medicamento y la terapia con fluidos, pero puede llegar progresar a insuficiencia renal crónica (8). La necesidad de hemodiálisis es rara y las medidas de prevención incluyen evitar administración de bolos en infusión rápida, aumentar el volumen de dilución del medicamento y ajustar la dosis a la función renal del paciente (7).

Las alteraciones en el estado de consciencia que se presentan en pacientes que reciben diferentes fármacos deben ser estudiadas en una forma sistemática. En esta paciente los cambios en el estado neurológico podrían haber sido producidos por efecto directo de cualquiera de los fármacos que estaba recibiendo. Sin embargo, inicialmente fueron atribuidos a los opioides y sólo hasta que fueron suspendidos (24 horas) se estableció el diagnóstico de encefalopatía urémica. La administración de aciclovir en pacientes con función renal alterada puede causar neurotoxicidad por sí mismo, con manifestaciones neuropsiquiátricas incluyendo alucinaciones y coma (6). En esta paciente no se realizaron mediciones de metabolitos opioides por la no disponibilidad en la institución, pero es posible que hubieran contribuido en el deterioro del estado neurológico. Hay reportes de casos en donde se encuentra que las manifestaciones de intoxicación por metabolitos activos de la morfina pueden continuar aun después de la hemodiálisis en pacientes con insuficiencia renal (9).

Shodi y Ratan en el año 2003 reportaron un caso de nefrotoxicidad con la administración oral de antiviral (8) lo que debe alertar sobre la monitorización de la función renal y síntomas neurológicos en los pacientes en manejo ambulatorio con aciclovir y otras drogas de eliminación renal.

\section{CONCLUSIONES}

Con frecuencia nos encontramos con situaciones en donde se presenta alteración del estado de consciencia en pacientes que reciben opioides, y es común que se piense que la causa de dicha alteración sea un efecto directo de estos medicamentos. Consideramos importante la monitorización de la función renal en los pacientes que presenten factores de riesgo para el desarrollo de nefrotoxicidad por antivirales como son la edad avanzada, la insuficiencia renal previa y el estado de deshidratación, con la finalidad de no retrasar el diagnóstico y brindar un tratamiento oportuno. 


\section{CORRESPONDENCIA:}

Carlos Guerrero

Departamento de Anestesiología y Clínica de Dolor

Hospital Universitario Fundación Santafé de Bogotá

Colombia

e-mail: carlosguerreromd@me.com

\section{BIBLIOGRAFÍA}

1. Genc G, Ozkaya O, Acikgöz Y, Yapici O, Bek K, Gülnar Sensoy $\mathrm{S}$, et al. Acute renal failure with acyclovir treatment in a child with leukemia. Drug Chem Toxicol 2010;33(2):217-9.

2. Obada EN, Level G, Mathieu P, Parent X, Gilson B, Bindi $\mathrm{P}$. Acute renal failure following a treatment withacyclovir. Nephrol Ther 2010;6(2):125-7.

3. De Deyne S, De la Gastine B, Gras G, Dargère S, Verdon R, Coquerel A. Acute renal failure with acyclovir in a 42-yearold patient without previous renal dysfunction. Rev Med Interne 2006;27(11):892-4.
4. Seedat A, Winnett G. Acyclovir-induced acute renal failure and the importance of an expanding waist line. BMJ Case Rep 2012;2012. doi: 10.1136/bcr-2012-006264.

5. Schreiber R, Wolpin J, Koren G. Determinants of aciclovir-induced nephrotoxicity in Children. Paediatr Drugs 2008;10(2):135-9.

6. Skhiri H, Achour A, Skhiri S, Frih A, Bouraoui S, Dhia NB, et al. Neuropsychiatric manifestations in a patient undergoing hemodialysis caused by treatment with oral acyclovir. Saudi J Kidney Dis Transpl 2004;15(1):50-2.

7. Pacheco LR, Tavares HM, Moysés Neto M, Dantas M, Rocha LS, Ribeiro KM, et al. Acute renal failure related to intravenous acyclovir. Rev Assoc Med Bras 2005;51(5):2758.

8. Sodhi PK, Ratan SK. A case of chronic renal dysfunction following treatment with oral acyclovir. Scand J Infect Dis 2003;35(10):770-2.

9. Izumi N. Prolonged disturbance of consciousness and respiratory depression induced by controlled-release morphine, requiring long-term naloxone administration in a hemodialysis patient with cancer: A case report. Gan To Kagaku Ryoho 2012;39(8):1295-9. 